\title{
Ocorrência de sangramento gengival na primeira infância em clínica integrada infantil de uma instituição de ensino superior
}

\author{
Occurrence of gingival bleeding in early childhood in a children's integrated clinico of a higher \\ education institution
}

Presencia de sangrado gingival em la primera infancia en una clínica infantil integrada de una institución de educación superior

\author{
Maísa Sales Oliveira e Silva \\ ORCID: https://orcid.org/0000-0002-6321-3471 \\ Centro Universitário de João Pessoa, Brasil \\ E-mail: maisasareis0425@gmail.com \\ Bianca Maria de Melo Costa \\ ORCID: https://orcid.org/0000-0002-4825-9420 \\ Centro Universitário de João Pessoa, Brasil \\ E-mail: biancamaria_pb@hotmail.com \\ Lucas Santana Cardoso \\ ORCID: https://orcid.org/0000-0002-7673-2342 \\ Centro Universitário de João Pessoa, Brasil \\ E-mail: lucassantanac21@gmail.com \\ Bianne Maria de Melo Costa \\ ORCID: https://orcid.org/0000-0002-2323-3072 \\ Centro Universitário de João Pessoa, Brasil \\ E-mail: biannemaria@hotmail.com \\ Bianca Oliveira Torres \\ ORCID: https://orcid.org/0000-0002-7256-2577 \\ Centro Universitário de João Pessoa, Brasil \\ E-mail: biancaotorres@yahoo.com.br \\ Roberta Remiliana Silva Trinta \\ ORCID: https://orcid.org/0000-0003-1868-9400 \\ Centro Universitário de João Pessoa, Brasil \\ E-mail: roberta.trinta@unipe.edu.br
}

\begin{abstract}
Resumo
O estudo teve como objetivo verificar a prevalência de sangramento gengival em crianças na primeira infância em uma clínica infantil de Odontologia, visando salientar a importância da promoção a saúde bucal e buscando subsidiar os profissionais de Odontologia a diagnosticar e ajudar a prevenir a gengivite em crianças. Trata-se de um estudo transversal, descritivo e quantitativo, realizado nas dependências da Clínica-Escola de Odontologia do Centro Universitário de João Pessoa (UNIPÊ). A amostra foi obtida, de forma não probabilística por conveniência, sendo constituída de 100 prontuários de crianças de 3 a 6 anos. As informações foram registradas em uma ficha para tratamento estatístico dos dados. Em relação ao sexo, 53\% das crianças eram do sexo masculino e $47 \%$ feminino. A ocorrência do sangramento gengival em crianças da primeira infância foi observada em $63 \%$ da amostra e $37 \%$ não apresentaram nenhum sangramento gengival. Em $49 \%$ das crianças obteve-se o escore do Índice de Sangramento Gengival (ISG) entre 1 a $10 \%$; em $11 \%$ obteve-se o escore do ISG entre 11 a $25 \%$ e apenas $3 \%$ obteve o escore do ISG entre 26 e $50 \%$. Em relação a presença de biofilme, as crianças de cinco e seis anos apresentaram uma porcentagem maior do Índice de Higiene Bucal (IHB), assim como ISG. Concluiu-se que a ocorrência do sangramento gengival nas crianças da primeira infância desta pesquisa foi elevada (63\%), não tendo sua ocorrência influenciada pelo sexo, mas associada ao aumento de idade. A presença do sangramento mostrou-se associada ao acúmulo do biofilme.
\end{abstract}

Palavras-chave: Gengivite; Crianças; Odontopediatria; Primeira infância.

\begin{abstract}
The study aimed to verify the prevalence of gingival bleeding in children in early childhood at a children's dental clinic, aiming to highlight the importance of promoting oral health and seeking to subsidize dental professionals to diagnose and help prevent gingivitis in children. This is a cross-sectional, descriptive and quantitative study, carried out on the premises of the Clínica-Escola de Odontologia of Centro Universitário de João Pessoa (UNIPÊ). The sample was obtained, in a non-probabilistic way for convenience, consisting of 100 records of children aged 3 to 6
\end{abstract}


years. The information was recorded on a form for statistical treatment of the data. Regarding gender, $53 \%$ of the children were male and $47 \%$ female. The occurrence of gingival bleeding in early childhood children was observed in $63 \%$ of the sample and $37 \%$ did not present any gingival bleeding. In $49 \%$ of the children, the Gingival Bleeding Index (ISG) score was between 1 and 10\%; in 11\% the ISG score was obtained between 11 to $25 \%$ and only $3 \%$ obtained the ISG score between 26 and 50\%. Regarding the presence of biofilm, children aged five and six years had a higher percentage of the Oral Hygiene Index (IHB), as well as ISG. It was concluded that the occurrence of gingival bleeding in early childhood children in this research was high (63\%), with its occurrence not influenced by sex, but associated with increasing age. The presence of bleeding was shown to be associated with the accumulation of biofilm.

Keywords: Gingivitis; Kids; Pediatric dentistry; Early childhood.

\section{Resumen}

El estudio tuvo como objetivo verificar la prevalencia de sangrado gingival en niños en la primera infancia en una clínica dental infantil, con el objetivo de resaltar la importancia de promover la salud bucal y buscar subsidiar a los profesionales dentales para diagnosticar y ayudar a prevenir la gingivitis en niños. Se trata de un estudio transversal, descriptivo y cuantitativo, realizado en las instalaciones de la Clínica-Escola de Odontologia del Centro Universitário de João Pessoa (UNIPÊ). La muestra se obtuvo, de forma no probabilística por conveniencia, compuesta por 100 registros de niños de 3 a 6 años. La información se registró en un formulario para el tratamiento estadístico de los datos. En cuanto al género, el 53\% de los niños eran varones y el $47 \%$ mujeres. La ocurrencia de sangrado gingival en niños de la primera infancia se observó en el 63\% de la muestra y el $37 \%$ no presentó sangrado gingival. En el $49 \%$ de los niños, la puntuación del índice de sangrado gingival (ISG) estaba entre 1 y 10\%; en el 11\% la puntuación ISG se obtuvo entre el 11 y el $25 \%$ y solo el $3 \%$ obtuvo la puntuación ISG entre el 26 y el $50 \%$. En cuanto a la presencia de biofilm, los niños de cinco y seis años presentaron mayor porcentaje del Índice de Higiene Bucal (BHI), así como del ISG. Se concluyó que la ocurrencia de sangrado gingival en niños de la primera infancia en esta investigación fue alta (63\%), y su ocurrencia no fue influenciada por el sexo, pero asociada con el aumento de edad. Se demostró que la presencia de sangrado está asociada con la acumulación de biopelícula.

Palabras clave: Gingivitis; Niños; Odontología pediatrica; Nlñez temprana.

\section{Introdução}

As doenças da cavidade oral são tão arcaicas quanto a própria humanidade, toda via, levantamentos epidemiológicos das condições de saúde bucal só tiveram início há algumas décadas, visando o conhecimento das tipologias das doenças e sua prevalência. Foi em um estudo experimental em humanos, realizado por Löe, Theilade e Jensen (1965) que se observou que o acúmulo de biofilme acarretava o desenvolvimento de uma doença que afeta os tecidos de proteção dos dentes, causando inflamação destes tecidos, levando a um quadro de gengivite. Observou também que remoção do biofilme, fator determinante, resultava numa melhora do quadro da inflamação gengival. Estes tipos de informações sistemáticas tornaram-se um instrumento importante no entendimento das determinantes que causam as doenças em 5 indivíduos com características semelhantes, contribuindo, assim, para os métodos de prevenção e tratamento de inúmeras doenças (Chambrone, Macedo, Ramalho, Trevizani Filho \& Chambrone, 2010).

A idade é um fator não modificável, independentemente se o paciente é pediátrico ou adulto o que vai dizer o grau da doença, se houver, são os fatores modificadores, como por exemplo: fatores de retenção de biofilme, restaurações defeituosas, restaurações mal adaptadas ou até mesmo com sobrecontorno. A maior prevalência dessa doença em indivíduos idosos reflete, apenas, o tempo em que os fatores locais permaneceram na superfície dentária e, pelo seu efeito cumulativo, provocaram a resposta inflamatória dos tecidos periodontais. A criança, à semelhança do adulto, é suscetível às doenças periodontais e, em especial, às gengivites que muitas vezes é uma resposta a má higiene bucal. Esse problema tem sido intensivamente estudado, em todo o mundo, nas últimas duas décadas. No Brasil, por exemplo, a média da prevalência de gengivite em crianças de 7 a 12 anos é $90 \%$ (Guedes-Pinto, 2016).

Os índices têm suma importância na saúde como um todo, pois através deles podemos medir, mensurar, atribuir valores para criar padrões, assim permitindo facilitar tanto o diagnóstico quanto à comunicação entre profissionais. Os índices surgiram com os sanitaristas que tinham e têm o ideal de promoção da saúde, bem-estar e qualidade de vida. Para isso, eles lançam mão de uma pesquisa bastante abrangente, denominada de análise epidemiológica (Greene \& Vermillion, 1960). 
É evidente que um paradoxo está ocorrendo; sabe-se qual é o fator determinante da doença periodontal, biofilme, os recursos para o seu controle são eficientes, higiene bucal e, mesmo assim, a prevalência de doença periodontal não está diminuindo. Parece lógico concluir que os recursos de prevenção não vêm sendo utilizados adequadamente em saúde pública e, muito provavelmente, em consultório particular (Guedes-Pinto, 2016).

A primeira infância é um período fundamental no desenvolvimento psicossocial do indivíduo e a promoção de saúde bucal para crianças abaixo de cinco anos é essencial para manter a saúde e o desenvolvimento adequado da digestão, fonação e respiração. A idade da primeira visita preventiva ao dentista tem um efeito positivo, o tratamento preventivo além de promover saúde bucal, também ajudará a diminuir os gastos com tratamento odontológico. Os dentes decíduos, servem como um guia para a dentição permanente, ou seja, a perda precoce desses elementos pode resultar em problemas de oclusão, por exemplo. Com isso, é muito relevante manter os cuidados de higiene e consultas com o dentista em qualquer idade (Alves et al., 2017).

Dessa forma, é de suma importância o exame periodontal em crianças e em conjunto com ele, seja feita a instrução de higiene oral e motivação, principalmente para o responsável pela educação da criança, tendo em vista a falta de maturidade nessa idade para discernir a seriedade do autocuidado. Assim, o objetivo desse trabalho é verificar a prevalência de sangramento gengival na primeira infância, e, com isto, salientar a importância da promoção de saúde.

\section{Metodologia}

Trata-se de um estudo transversal, descritivo e quantitativo. Os estudos epidemiológicos podem ser classificados em observacionais e experimentais, sendo, esse trabalho, observacional. De uma maneira geral, os estudos epidemiológicos observacionais podem ser classificados em descritivos e analíticos. Uma de suas peculiaridades está na utilização de técnicas padronizadas de coleta de dados, como a ficha de registro. O estudo descritivo tem por objetivo determinar a distribuição de doenças ou condições relacionadas à saúde, segundo o tempo, o lugar e/ou as características dos indivíduos (Lima-Costa \& Barreto, 2003).

A pesquisa tem aspecto quantitativo que, para Fonseca (2002), "diferentemente da pesquisa qualitativa, os resultados da pesquisa quantitativa podem se quantificados. Como as amostras geralmente são grandes e consideradas representativas da população, os resultados são tomados como se constituíssem um retrato real de toda a população alvo da pesquisa. A pesquisa quantitativa se centra na objetividade".

A pesquisa foi desenvolvida nas dependências da Clínica-Escola de Odontologia do Centro Universitário de João Pessoa (UNIPÊ), onde foram analisados os prontuários dos pacientes de 3 a 6 anos da Clínica Integrada Infantil I e II dos anos 2018 e 2019. Os valores percentuais do Índice de Sangramento Gengival (ISG) foram agrupados nos seguintes escores: sem sangramento (1); de 1 a $10 \%$ de faces com sangramento (2); de 11 a $25 \%$ (3); de 26 a $50 \%$ (4); de 51 a $75 \%$ (5); acima de $75 \%$ de faces com sangramento (6).

De acordo com Fachin (2003), o universo é o conjunto sobre cujos atributos vão indiciar a investigação e, por isso, se transformarão em fonte de informação. A população do estudo foi constituída por 100 prontuários de pacientes de três a seis anos atendidas na Clínica Integrada Infantil I e II nos anos 2018 e 2019. Importante salientar aqui que apesar da fase da primeira infância iniciar antes dos 3 anos, a clínica-escola só atende pacientes infantis a partir de três anos. A amostra não probabilística, obtida por conveniência correspondeu a 100 prontuários.

Os critérios de inclusão consistiram em prontuários de pacientes com idade entre três e seis anos, atendidos na Clínica-Escola de Odontologia do UNIPÊ, no período de 2018 e 2019; os prontuários devidamente preenchidos, especialmente que informaram o ISG e que apresentaram informações relativas aos objetivos da pesquisa. Já os critérios de exclusão foram os prontuários que apresentaram falta de informações ou rasuras que impediu a correta coleta de dados. 
O instrumento de coleta de dados desta pesquisa foi uma ficha de registros, onde foram anotadas informações coletadas dos prontuários das Clínicas Integradas Infantil I e II que verificaram a ocorrência de sangramento gengival.

Inicialmente foi encaminhado o projeto de pesquisa para o Comitê de Ética em Pesquisa do UNIPÊ (CEP/UNIPÊ). Em seguida, com a aprovação e certificação do CEP/UNIPÊ, foi realizado a solicitação dos prontuários aos responsáveis. O acesso aos prontuários foi feito apenas pelos pesquisadores. Em seguida foi coletadas as informações necessárias para esse trabalho e registradas em ficha de coleta.

De acordo com a Resolução CNS/MS 466/2012 (BRASIL, 2012), toda pesquisa oferece riscos de gradações diferentes. Os riscos previstos para essa pesquisa foram mínimos, uma vez que consistiu apenas em análise de fichas de registros com informações contidas em prontuários, respeitando-se os preceitos éticos como o sigilo. Para minimizar estes riscos, os pesquisadores comprometem-se em salvaguardar as identidades dos participantes, garantindo o anonimato, divulgando somente as informações consolidadas, tendo, apenas, os pesquisadores acesso aos prontuários.

A pesquisa respeitou os aspectos éticos da resolução 466/2012, que descreve as normas de pesquisa com seres humanos, tendo a coleta dos dados sido processada somente após a aprovação pelo Comitê de Ética e Pesquisa institucional através do Parecer no 4.192.212 e do CAAE n 35125120.3.0000.5176.

O presente estudo evidenciou a ocorrência de doenças periodontais em crianças da primeira infância e suas consequências, e trouxe como benefício a conscientização aos estudantes de Odontologia e cirurgiões-dentistas da importância do exame periodontal, promoção da saúde bucal, motivação do paciente, resultando numa melhoria da qualidade de vida do paciente.

\section{Resultados e Discussão}

O presente estudo, apresentou uma amostra não probabilística, obtida por conveniência. Foram analisados 100 prontuários, sendo 53\% do sexo masculino e $47 \%$ do sexo feminino. Em relação a faixa etária, 19 crianças com 3 anos; 21 delas com 4 anos; 25 tinham 5 anos e 35 com 6 anos (Gráfico 1).

Gráfico 1 - Distribuição da amostra pela idade.

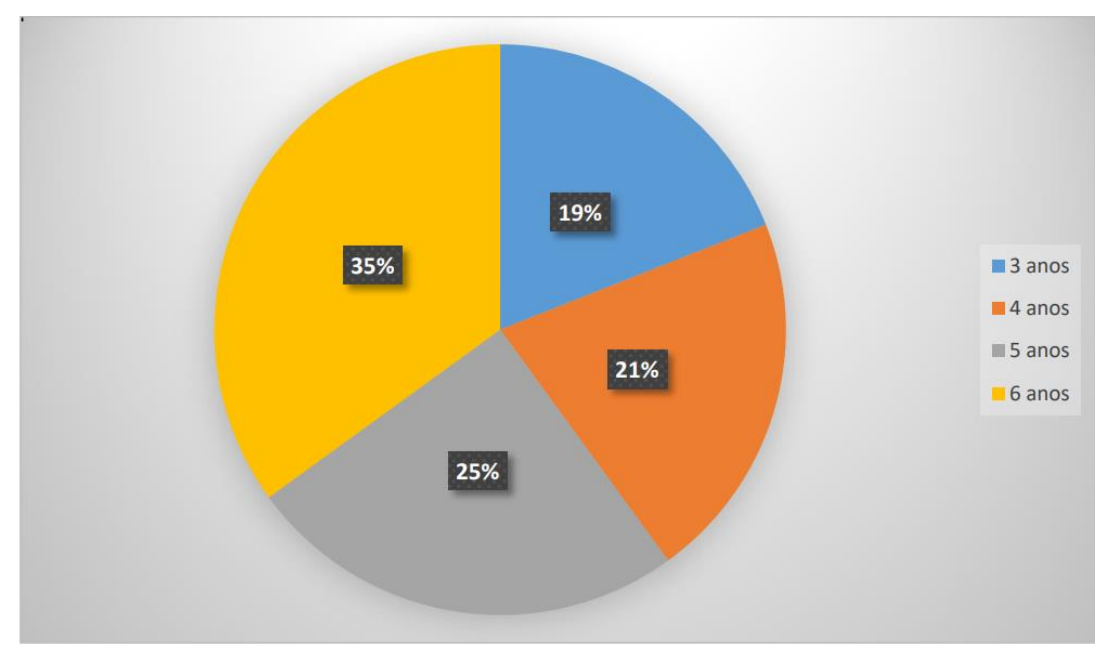

Fonte: Dados da Pesquisa (2020).

Em 63\% da amostra desta pesquisa, as crianças apresentaram sangramento gengival e 37 \% apresentaram ausência de sangramento. Divergindo do estudo feito por Teixeira (2006) que assistiu 960 crianças de escolas municipais em 2005, na cidade de Piracicaba no estado de São Paulo, observou-se que 68,3\% dos integrantes da pesquisa apresentaram todos os sextantes sadios e que o $31,7 \%$ apresentou algum sextante com sangramento. 
Foi observado sangramento gengival em 33 crianças do sexo masculino e 30 crianças do sexo feminino, enquanto que 37 crianças não apresentaram sangramento, o que demonstra nesta amostra falta de associação entre doença gengival e sexo (Tabela 1).

Tabela 1 - Distribuição do Sangramento Gengival por sexo.

\begin{tabular}{l|c|c}
\hline Sexo & n & \% \\
\hline Masculino & 33 & 52,4 \\
\hline Feminino & 30 & 47,6 \\
\hline Total & 63 & $100 \%$ \\
\hline
\end{tabular}

Fonte: Dados da Pesquisa (2020).

Em relação a ocorrência do sangramento gengival por idade foi observado em 10 crianças com 3 anos; 11 crianças com 4 anos; 17 crianças com 5 anos e 25 crianças com 6 anos (Tabela 2). Assim, foi percebido que a presença de sangramento gengival estava relacionada com o aumento da idade. Neste aspecto corroborando com Teixeira (2006), em que aos 3 anos a percentagem de crianças com todos os sextantes sadios foi de $77,2 \%$, enquanto que aos 6 anos essa porcentagem diminuiu para $63,3 \%$

Tabela 2 - Distribuição do Sangramento Gengival por idade.

\begin{tabular}{l|c|c}
\hline Idade & n & \% \\
\hline 3 anos & 10 & $53 \%$ \\
\hline 4 anos & 11 & $52,4 \%$ \\
\hline 5 anos & 17 & $68 \%$ \\
\hline 6 anos & 25 & $71,4 \%$ \\
\hline
\end{tabular}

Fonte: Dados da Pesquisa (2020).

Em relação ao percentual de faces sangrantes do ISG desta pesquisa (escores 1 ao 6 do ISG), constatou-se que 37\% das crianças não tiveram sangramento gengival, $49 \%$ das crianças obtiveram um ISG de 1 a $10 \%, 11 \%$ obtiveram o ISG de 11 a 25\% e apenas 3 obtiveram o ISG de 26 a 50\% (Tabela 3). Divergindo do estudo realizado por Moraes e Valença (2003) na cidade de Aracaju (SE), foram examinadas 518 crianças com idade de 3 a 5 anos. Obteve-se o resultado de que a gengivite acometeu respectivamente, 74,5\% ( $\mathrm{n}=76), 78,2 \%(\mathrm{n}=197)$ e 72\% (n=118), dos pré-escolares de 3, 4 e 5 anos. Eles também observaram que $24,5 \%$ não tiveram sangramento gengival; a maior parte, $68 \%$ obtiveram um ISG de 1 a $10 \%$ e $7 \%$ obtiveram um ISG de 11 a $25 \%$, divergindo desta pesquisa, nenhuma criança obteve ISG maior que $26 \%$.

Notou-se também uma associação entre o nível de higiene oral e a prevalência de gengivite, sendo esta alteração significativamente menos frequente nas crianças com higiene oral satisfatória. Corroborando com o estudo feito por Demari, Marques, Pereira, Oliveira e Werle (2016), com uma amostra menor, 55 crianças entre zero a 6 anos de idade que procuraram atendimento na Clínica de Odontopediatria da Faculdade da Serra Gaúcha (FSG), Caxias do Sul-RS, Brasil, entre os meses de outubro de 2014 e maio de 2015, foi constatado que 76, $1 \%$ da amostra apresentaram gengivite, 36 crianças, nesse estudo, apresentaram um Índice de Sangramento Gengival menor que 20\% e 14 delas apresentaram um Índice de Sangramento maior que 20\%. Demari et al. (2016) enfatizou que o nível de conhecimento dos pais foi considerado limitado, em relação à higiene bucal, e a capacidade de controle de biofilme da criança não foi adequada, o que contribuiu para o desenvolvimento da gengivite. 
Tabela 3 - Distribuição do ISG segundo a porcentagem de faces acometidas pela gengivite nas crianças avaliadas na ClínicaEscola do UNIPÊ.

\begin{tabular}{l|c|c}
\hline Escores de ISG & $\mathbf{n}$ & \% \\
\hline Sem sangramento & 37 & $37 \%$ \\
\hline 1 a $10 \%$ & 49 & $49 \%$ \\
\hline 11 a $25 \%$ & 11 & $11 \%$ \\
\hline 26 a $50 \%$ & 3 & $3 \%$ \\
\hline
\end{tabular}

Fonte: Dados da Pesquisa (2020).

Na presente pesquisa não houve diferença significativa no Índice de Sangramento Gengival (ISG) das crianças do sexo masculino e feminino. Divergindo do estudo feito por Cortellazi (2006), na cidade de Piracicaba-SP, que teve uma amostra com 728 crianças, com a idade de cinco anos do sexo feminino e masculino. O resultado da pesquisa de Cortellazi (2006), foi que o percentual de crianças na faixa etária de cinco anos que apresentaram ausência e presença de sangramento gengival foi de $83,3 \%$ e $16,6 \%$, respectivamente, sendo a presença de sangramento gengival mais prevalente nas crianças do sexo masculino.

Granville-Garcia, Ferreira, Barbosa, Vieira, Siqueira e Menezes (2010) realizaram um estudo de prevalência em 820 crianças com idade de 1 a 5 anos, no município de Caruaru-PE, em 2007. A determinação da gengivite foi realizada por meio do exame visual, no qual foram verificadas alterações de cor e forma da gengiva. A prevalência de gengivite foi de 10,9\% entre as cinco faixas etárias que foram analisadas, pode-se sugerir que este percentual foi minimizado, devido ao método de critério ser somente visual. Também não se comprovou associação significante entre idade e ocorrência de gengivite. Já Soares, Aragão, Jardim e Sampaio (2003), que tiveram como objetivo relacionar a gengivite com o IHB de 120 escolares com idade de 7 a 12 anos, realizada no município de Cabedelo-PB, utilizaram a análise visual da gengiva (cor, textura, formato) e o ISG como parâmetros de diagnóstico da gengivite. Obteve-se os seguintes resultados: a gengivite através do aspecto clínico foi diagnosticada em 40,8\% da amostra. Já utilizando-se o ISG, notou-se uma prevalência de $85 \%$ de gengivite. O IHB médio foi de 1,96, sendo a correlação da prevalência e severidade da gengivite com o IHB foi significativa.

Em relação ao IHB, obteve-se nos resultados da presente pesquisa que as crianças com idade de 3 e 4 anos apresentaram um percentual maior de nível satisfatório (escore 0-1), por outro lado, as crianças de 5 e 6 anos apresentaram um percentual maior de nível regular (escore 1-2) e um menor percentual de nível satisfatório. O sexo masculino e feminino se manteve praticamente com a mesma porcentagem no IHB (Tabela 4). Divergindo da pesquisa de Teixeira (2006), na qual as crianças com idade de cinco e seis anos apresentaram maior percentual de nível baixo de biofilme, enquanto as de três e quatro apresentaram maiores percentagens de nível moderado e alto. Teixeira (2006) afirmou que a maioria dos pré-escolares examinados na sua pesquisa $81,1 \%$, apresentou nível baixo $81,1 \%$ (escores: 0 - 1), enquanto que $18,2 \%$ e $0,7 \%$ apresentaram nível moderado (escores: 1-2) ou alto (escores: 2-3). 
Tabela 4 - Distribuição do IHB pelo sexo e idade das crianças avaliadas na Clínica-Escola do UNIPÊ.

\begin{tabular}{c|c|c|c|c|c|c}
\hline & \multicolumn{2}{|c|}{ Satisfatório } & \multicolumn{2}{c|}{ Deficiente } & \multicolumn{2}{c}{ Regular } \\
\hline Sexo & $\mathbf{n}$ & $\boldsymbol{\%}$ & $\mathbf{n}$ & $\mathbf{\%}$ & $\mathbf{n}$ & \% \\
\hline Masculino & 10 & $18,9 \%$ & 20 & $37,7 \%$ & 23 & $43,4 \%$ \\
\hline Feminino & 8 & $17,1 \%$ & 18 & 38,3 & 21 & $44,7 \%$ \\
\hline Total & 18 & $36 \%$ & 38 & $70 \%$ & $44 \%$ & 88,1 \\
\hline & \multicolumn{2}{|c|}{ Satisfatório } & \multicolumn{2}{|c|}{ Deficiente } & \multicolumn{2}{c}{ Regular } \\
\hline Idade & $\mathbf{n}$ & $\boldsymbol{\%}$ & $\mathbf{n}$ & $\mathbf{\%}$ & $\mathbf{n}$ & \% \\
\hline 3 anos & 8 & $42,0 \%$ & 5 & $26,4 \%$ & 6 & $31,6 \%$ \\
\hline 4 anos & 9 & 42,8 & 6 & $28,6 \%$ & 6 & $28,6 \%$ \\
\hline 5 anos & 6 & $24 \%$ & 7 & $28 \%$ & 12 & $48 \%$ \\
\hline 6 anos & 9 & $25,8 \%$ & 11 & $31,4 \%$ & 15 & $42,8 \%$ \\
\hline
\end{tabular}

Fonte: Dados da Pesquisa (2020).

Para Sharma e Galustians (1994), a escovação é a linha de frente de defesa contra o biofilme dental e a gengivite, ou seja, ao prevenir o biofilme, estaremos evitando a gengivite, pois é a sua principal causa. Esse fato foi documentado nos estudos clássicos de Löe et al. (1965), onde a interrupção dos procedimentos de escovação dental resultou em rápido acúmulo do biofilme dental e desenvolvimento de gengivite num período de três semanas. A higienização bucal deve ser ainda mais rigorosa em crianças, ajudando e instruindo-as até conseguirem a habilidade necessária para realizar sua própria higienização, a motivação deve ser feita pelos pais e pelos profissionais de saúde através da promoção à saúde bucal, assim a criança criará bons hábitos de higiene bucal e, com isto, estará se prevenindo das diversas doenças bucais.

\section{Considerações Finais}

Conclui-se que a ocorrência do sangramento gengival nas crianças da primeira infância desta pesquisa foi elevada (63\%), não tendo sua ocorrência influenciada pelo sexo, mas associada ao aumento de idade, principalmente nas crianças de cinco e seis anos, verificando-se a ocorrência de sangramento gengival de leve a moderada, não tendo sido registrados casos de inflamação gengival severa. A presença do sangramento mostrou-se associada ao acúmulo do biofilme, IHB da amostra mostrou-se $70 \%$ deficiente e $88,1 \%$ regular, ficando apenas 36\% com escore satisfatório.

Dessa forma, cabe aos profissionais e aos responsáveis da criança orientar, manter, auxiliar e supervisionar a escovação regular, de maneira que ela seja realizada da forma correta e que os quadros de inflamação gengival sejam diagnosticados e revertidos rapidamente, e não postergado.

Sabendo-se da necessidade dos cuidados bucais, não apenas com a estrutura dentária, mas também com o periodonto, é importante que trabalhos futuros sejam realizados com amostras maiores para ratificar os achados dessa pesquisa, além de buscas de alternativas e incentivos que proporcionem melhorias na qualidade de higienização desse público, buscando dessa forma, estratégias que revertam o quadro de inflamação gengival.

\section{Referências}

Alves, A. P. S., Rank, R. C. I. C., Vilela, J. E. R., Ogawa, W. N. \& Molina, O. F. (2018). Efficacy of a public promotion program on children's oral health. Jornal De Pediatria, 94(5), 518-524, 2018. http://search.ebscohost.com/login.aspx?direct=true \&db=mdc\&AN=28958799\&authtype=sso $\&$ custid=ns000688\&lang=pt-br\&site=eds-live \&scope $=$ site.

Brasil, Ministério da Saúde. Resolução $\mathrm{n}^{\circ}$ 466, de 12 de dezembro de 2012. https://bvsms.saude.gov.br/bvs/saudelegis/cns/2013/res0466 _12_12_2012.html\#: :text=Toda\%20pesquisa\%20com\%20seres\%20humanos,Sistema\%20CEP\%2FCONEP\%20aos\%20participantes. 
Research, Society and Development, v. 10, n. 1, e36510111837, 2021

(CC BY 4.0) | ISSN 2525-3409 | DOI: http://dx.doi.org/10.33448/rsd-v10i1.11837

Chambrone, L., Macedo, S. B., Ramalho, F. C., Trevizani Filho, E. \& Chambrone, L. A. (2010). Prevalência e severidade de gengivite em escolares de 7 a 14 anos: Condições locais associadas ao sangramento à sondagem. Ciência e Saúde Coletiva, 15(2), 337-343. https://www.scielosp.org/article/csc/2010.v15n2/337-343/pt/.

Cortellazzi, k. 1. (2006). Indicadores de risco de cárie dentária e de gengivite em crianças na faixa etária de 5 anos (Dissertação de mestrado). Universidade Estadual de Campinas, Faculdade de Odontologia de Piracicaba, Piracicaba, SP, Brasil. http://repositorio.unicamp.br/jspui/handle/REPOSIP/290335.

Demari, S., Marques, C., Pereira, J.T., Oliveira, R.S. \& Werle S.B. (2016). Avaliação do conhecimento sobre higiene bucal dos responsáveis por crianças de 06 anos de idade. Revista da Faculdade de Odontologia de Lins, 26(1), 11-18. https://www.metodista.br/revistas/revistasunimep/index.php/Fol/article/view/2777.

Fachin, O. (2003). Fundamentos de metodologia (4a ed.). Saraiva.

Fonseca, J. J. S. (2002). Metodologia da pesquisa científica. Fortaleza: UEC. http://www.ia.ufrrj.br/ppgea/conteudo/conteudo-20121/1SF/Sandra/apostilaMetodologia.pdf.

Granville-Garcia, A. F., Ferreira, J. M. S., Barbosa, A. M. F., Vieira, I., Siqueira, M. J. \& Menezes, V. A. (2010). Cárie, gengivite e higiene bucal em préescolares. Revista Gaúcha de Odontologia, 58(4). http://revodonto.bvsalud.org/scielo.php?script=sci_arttext\&pid=S1981-86372010000400007.

Greene, J. C. \& Vermillion, J. R. (1960). The oral hygiene index: a method of classifying oral hygiene status. J Amer dent Ass, 61, 172-9. https://www.sciencedirect.com/sdfe/pdf/download/eid/1-s2.0-S0002817760120037/first-page-pdf.

Guedes-Pinto, A. C. (2016). Manual de Odontopediatria (9a ed.). Pancast.

Lima-Costa, M. F. \& Barreto, S.M. (2003). Tipos de estudos epidemiológicos: conceitos básicos e aplicações na área do envelhecimento. Epidemiol. Serv. Saúde, 12(4), 189-201. http://scielo.iec.gov.br/scielo.php?script=sci_arttext\&pid=S1679-49742003000400003\&lng=pt\&nrm=iso.

Löe, H., Theilade, E. \& Jensen, S. B. (1965). Experimental gingivitis in man. $J$ Periodontol., 36, 177-187. https://aap.onlinelibrary. wiley.com/doi/abs/10.1902/jop.1965.36.3.177.

Moraes, E. S. \& Valença, A.M.G. (2003). Prevalência de gengivite e periodontite em crianças de 3 a 5 anos na cidade de Aracajú. Cienc Odontol Bras., 6(4), 87-94. http://bds.ict.unesp.br/index.php/cob/article/view/580/490.

Sharma, N.C. \& Galustians, J. (1994). Efeitos clínicos sobre a placa dental e a gengivite obtidos com o uso de quatro escovas dentais manuais de desenho complexo por período de três meses. J Clin Dentistr., 5, 114-8.

Soares, J. M. P., Aragão, A. K. R., Jardim, M. C. A. M. \& Sampaio, F. C. (2003). Gengivite e Índice de Higiene Oral em Crianças de Cabedelo - Paraíba Brasil. Ver. Bras. Ciênc. Saúde, 7(2), 177-186.

Texeira, M. S. (2006). Condição gengival em pré-escolares de 03 a 06 anos da rede municipal de ensino de Piracicaba-SP (Dissertação de Mestrado). Universidade Estadual de Campinas - Faculdade de Odontologia de Piracicaba, Piracicaba, SP, Brasil. http://repositorio.unicamp.br/jspui/bitstream/REPOSIP/333764/1/Teixeira_MilenaSchaaf_M.pdf. 